\title{
A GLOBAL EXISTENCE THEOREM FOR A NONAUTONOMOUS DIFFERENTIAL EQUATION IN A BANACH SPACE
}

DAVID LOWEI.L. LOVELADY AND ROBERT H. MARTIN, JR.

\begin{abstract}
Suppose that $X$ is a real or complex Banach space and that $A$ is a continuous function from $[0, x) \times X$ into $X$. Suppose also that there is a continuous real valued function $\rho$ defined on $[0, \infty)$ such that $A(t, \cdot)-\rho(t) I$ is dissipative for each $t$ in $[0, \infty)$. In this note we show that, for each $z$ in $X$, there is a unique differentiable function $u$ from $[0, \infty)$ into $X$ such that $u(0)=z$ and $u^{\prime}(t)=A(t, u(t))$ for all $t$ in $[0, \infty)$. This is an improvement of previous results on this problem which require additional conditions on $A$.
\end{abstract}

Let $X$ be a real or complex Banach space and let $|\cdot|$ denote the norm on $X$. It is the purpose of this note to prove the following theorem.

THEOREM. Suppose that $A$ is a continuous function from $[0, \infty) \times X$ into $X$ and that $\rho$ is a continuous real-valued function on $[0, \infty)$. Suppose also that

$$
|x-y-h[A(t, x)-A(t, y)]| \geqq(1-h \rho(t))|x-y|
$$

for each $(t, x, y)$ in $[0, \infty) \times X \times X$ and $h>0$. Then for each $z$ in $X$ there is a unique continuously differentiable function $u_{z}$ from $[0, \infty)$ into $X$ such that

$$
\begin{aligned}
& u_{z}(0)=z \quad \text { and } \\
& u_{z}^{\prime}(t)=A\left(t, u_{z}(t)\right)
\end{aligned}
$$

for all $t$ in $[0, \infty)$.

Condition (1) of the theorem implies that, for each $t$ in $[0, \infty)$, the function $x \rightarrow A(t, x)-\rho(t) x$ is dissipative on $X$. Also, if $m[x, y]=$ $\lim _{h \rightarrow 0-0}(|x+h y|-|x|) / h$ for each $(x, y)$ in $X \times X$. then condition (1) is easily seen to be equivalent to requiring that the inequality

$$
m[x-y, A(t, x)-A(t, y)] \leqq \rho(t)|x-y|
$$

is valid for all $(t, x, y)$ in $\left[0, x_{)}\right) \times X \times X$.

Received by the editors September 17, 1971 and, in revised form, January 27, 1972. AMS 1969 subject classifications. Primary 3495; Secondary 3404.

Key nords and phrase's. Nonautonomous differential equations, dissipative operators, global existence theorems, an application of nonlinear semigroups. 
Local existence and uniqueness of functions $u_{z}$ satisfying (2) and (3) follows from D. I. Lovelady [5]. Under supplementary assumptions on $A$, results analogous to those of the above theorem have been proved by several authors (R. H. Martin [6], $A$ independent of $t$, J. V. Herod [3] and G. F. Webb [8], $A$ bounded on bounded subsets of $[0, \infty) \times X$; and G. F. Webb [9] and R. H. Martin [7], $A(t,)^{\circ}$ satisfying an equicontinuity condition for $t$ in bounded subsets of $[0, \infty)$ ). The main point of this note is to show that global existence is assured without any additional assumptions. Our approach is different from those of the above cited results. In essence, we use a recent result of G. F. Webb [10] to show that the zero function is in the range of the operator which maps continuously differentiable $X$-valued functions $u$ on $[0, \infty)$ into the $X$-valued function $f$ on $[0, \infty)$ given by $f(t)=u^{\prime}(t)-A(t, u(t))$.

For the proof of the theorem, note that it suffices to show that for each $b>0$ there is a function $u_{z}$ satisfying (2) and (3) for all $t$ in $[0, b]$. Also, it is convenient to record the following elementary, properties of the function $m$ :

$$
m[\alpha x, \beta y]=\beta m[x, y] \text { for } x, y \in X \text { and } \alpha, \beta>0
$$

and

(6) $\quad m[x, y+\lambda x]=m_{-}[x, y]+\hat{\lambda}|x|$ for $x, y \in X$ and $\lambda$ real.

The following three lemmas are needed for the proof of the theorem. We assume that the suppositions of the theorem are fulfilled, that $b$ is a positive number, and that $\gamma^{\prime}=\max \{\rho(t)+1: t \in[0, b]\}$. Also, define

$$
B(t, x)=e^{-y t} A\left(t, e^{\gamma t} x\right)-\gamma x \text { for }(t, x) \in[0, \infty) \times X .
$$

The first lemma is immediate from the definition of $B$.

LEMmA 1. The function $B$ defined by (7) is continuous on $[0, \infty) \times X$, and there is a function $u_{z}$ satisfying (2) and (3) for $t$ in $[0, b]$ if and only if there is a function $v_{\text {. from }}[0, b]$ into $X$ satisfying $v_{z}(0)=z$ and $v_{z}^{\prime}(t)=B\left(t, v_{z}(t)\right)$ for each $t$ in [0,b]. Also, in this case, $u_{z}(t)=e^{\gamma t} v_{z}(t)$ for all $t$ in $[0, b]$.

Now let $\mathscr{t}$ denote the Banach space of all continuous functions $f$ from $(-\infty, b]$ into $X$ with $\lim _{t, \ldots \infty}|f(t)|=0$ and $\|f\|=\max \{|f(t)|: t \in(-\infty, b]\}$.

Lemma 2. Suppose that $D(L)=\left\{f \in \mathscr{C}: f^{\prime} \in \mathscr{C}\right\}$ and that $L[f]=-f^{\prime}$ for each $f$ in $D(L)$. Then $L$ is the generator of a strongly continuous linear contraction scmigroup on $[0, \infty)$.

INDICATION OI PRONF. It is straightforward to see that $L$ is a closed, densely defined linear operator on $\mathscr{C}$ (the fact that $L$. is closed follows from $[2,(8.6 .4), p .158])$, for example, and to see that $D(L)$ is dense; note 
the set of functions $g$ where $g(t)=\int_{-\infty}^{t} f(s) d s, f$ continuous with compact support, are in $D(L)$ and are dense in $\mathscr{C}$. Now suppose that $\lambda>0$ and $g$ is in $\mathscr{C}$. Define $f(t)=-e^{-\lambda t} \int_{-\infty}^{t} e^{\lambda s} g(s) d s$ for each $t$ in $(-\infty, b]$. Then $|f(t)| \leqq e^{-\lambda t} \int_{-\infty}^{t} e^{\lambda s}|g(s)| d s$; so $f \in \mathscr{C}$ (apply L'Hospital's Rule). Furthermore, $f$ is differentiable and $f^{\prime}=-g-\lambda f$; so $f \in D(L)$ and $(L-\lambda I)[f]=g$ (where $I$ is the identity mapping on $\mathscr{C}$ ). It now follows that $\lambda$ is in the resolvent of $L$ and, if $g$ is in $\mathscr{C}$,

$$
\begin{aligned}
\left\|(L-\lambda I)^{-1}[g]\right\| & \leqq \max \left\{e^{-\lambda t} \int_{-\infty}^{t} e^{\lambda s}|g(s)| d s: t \in:(-\infty, b]\right\} \\
& \leqq\|g\| \max \left\{e^{-\lambda t} \int_{-\infty}^{t} e^{\lambda s} d s: t \in(-\infty, b]\right\}=\|g\| \lambda^{-1} .
\end{aligned}
$$

Thus $L$ satisfies each of the conditions of the Hille-Yosida Theorem (see e.g. [4, Corollary, p. 363]), and the assertion of the lemma is true.

Lemma 3. Let $B$ be as in (7) and for each $f$ in $\mathscr{C}$ define the $X$-valued function $G[f]$ on $(-\infty, b]$ by $G[f](t)=B(t, f(t))$ if $t \in[0, b]$ and $G[f](t)=$ $e^{t} B(0, f(t))+\left(e^{t}-1\right) f(t)$ if $t \in(-\infty, 0)$. Then $G$ maps $\mathscr{C}$ into $\mathscr{C}, G$ is continuous on $\mathscr{C}$, and the inequality'

$$
\|f-g-h(G[f]-G[g])\| \geqq(1+h)\|f-g\|
$$

is valid for each of $f$ and $g$ in $\mathscr{C}$ and $h>0$.

INDICATION OF PROOF. Note first that $G[f]$ is continuous on $(-\infty, b]$. Since $\lim _{t \ldots \infty} B(0, f(t))=B(0,0)$, it easily follows that $G[f] \in \mathscr{C}$; so $G$ maps $\mathscr{C}$ into $\mathscr{H}$. Using the fact that the range of a member $f$ of $\mathscr{C}$ is a compact subset of $X$. for each $\varepsilon>0$ one can find a $\delta>0$ such that if $(t, x) \in$ $[0, b] \times X$ with $|f(t)-x| \leqq \delta$, then $|B(t, x)-B(t, f(t))| \leqq \varepsilon$, and if $(t, x) \in$ $(-\infty, 0) \times X$ with $|f(t)-x| \leqq \delta$, then $|B(0, x)-B(0, f(t))| \leqq \varepsilon$. The continuity of $G$ is now easily established. Now let $f$ and $g$ be in $\mathscr{f}$. Using (4), (5), (6) and the definitions of $G$ and $B$, we have that if $t \geqq 0$,

$$
\begin{aligned}
m_{-} & {[f(t)-g(t), G[f](t)-G[g](t)] } \\
& =m_{-}\left[f(t)-g(t), e^{-\gamma t} A\left(t, e^{\gamma t} f(t)\right)-e^{-\gamma t} A\left(t, e^{\gamma t} g(t)\right)\right]-\gamma|f(t)-g(t)| \\
& =e^{-\gamma t} m_{-}\left[e^{\gamma t} f(t)-e^{\gamma t} g(t), A\left(t, e^{\gamma t} f(t)\right)-A\left(t, e^{\gamma t} g(t)\right)\right]-\gamma|f(t)-g(t)| \\
& \leqq e^{-\gamma t} \rho(t)\left|e^{\gamma t} f(t)-e^{\gamma t} g(t)\right|-\gamma|f(t)-g(t)| \\
& =(\rho(t)-\gamma)|f(t)-g(t)| \leqq-|f(t)-g(t)|,
\end{aligned}
$$

by the definition of $\gamma$. Similarly, if $t<0$.

$$
\begin{aligned}
& m_{-}[f(t)-g(t), G[f](t)-G[g](t)] \\
& \quad=m_{-}\left[f(t)-g(t), e^{t} B(0, f(t))-e^{t} B(0, g(t))\right]+\left(e^{t}-1\right)|f(t)-g(t)| \\
& \quad \leqq e^{t}\left(\rho(0)-\gamma g|f(t)-g(t)|+\left(e^{t}-1\right)|f(t)-g(t)| \leqq-|f(t)-g(t)| .\right.
\end{aligned}
$$


Thus,

$$
m[f(t)-g(t), G[f](t)-G[g](t)] \leqq-|f(t)-g(t)|
$$

for all $t \in(-\infty), b]$. The inequality (8) is easily seen to imply that

$$
|f(t)-g(t)-h(G[f](t)-G[g](t))| \geqq(1+h)|f(t)-g(t)|
$$

for all $t \in(-\infty, b]$ and $h>0$. The final assertion of this lemma now follows by taking the supremum for $t$ in $(-\infty, b]$ of each side of the inequality (9).

Proof of Theorem. Let $g$ be a member of $\mathscr{C}$ and define $M[f]=$ $L[f]+G[f]-g$ for each $f$ in $D(L)$. Using Lemmas 2 and 3, we can apply Theorem II of G. F. Webb [10] to show that $M$ is the generator of a strongly continuous nonlinear semigroup $\{U(t): t \in[0, \infty)\}$ which satisfies $\left\|U(t) f_{1}-U(t) f_{2}\right\| \leqq e^{-t}\left\|f_{1}-f_{2}\right\|$ for all $\left(t, f_{1}, f_{2}\right)$ in $[0, \infty) \times \mathscr{C} \times \mathscr{C}$. Thus, for each $t>0, U(t)$ has a unique fixed point in $\mathscr{C}$, and since $U(t)$ and $U(s)$ commute, there is a unique $f_{g}$ in $\mathscr{C}$ such that $U(t) f_{g}=f_{g}$ for all $t \geqq 0$. Thus $f_{g} \in D(L)$ and

$$
M\left[f_{g}\right]=\lim _{t \rightarrow 0+} t^{-1}\left[U(t) f_{g}-f_{g}\right]=0 .
$$

It now follows that

$$
L\left[f_{g}\right]+G\left[f_{g}\right]=g .
$$

Now let $z$ be in $X$ and define $h(t)=c^{t} z+t e^{t}[B(0, z)-z]$ for each $t$ in $(-\infty, b]$. Then $h \in \mathscr{C}, h(0)=z, h^{\prime}(0)=B(0, z)$, and $\lim _{t \ldots \infty} h^{\prime}(t)=0$. Hence if $g(t)=0$ for $t \in[0, b]$, and $g(t)=-h^{\prime}(t)+G[h](t)$ for $t<0$, then $g \in \mathscr{C}$. If $f_{g}$ is as in (10), then

$$
f_{y}^{\prime}(t)=C\left[f_{g}\right](t)=B\left(t, f_{g}(t)\right) \text { if } t \in[0, b],
$$

and

$$
-f_{i i}^{\prime}(t)+G\left[f_{g}\right](t)=-h^{\prime}(t)+G[h](t) \quad \text { if } t \in(-\infty, 0] .
$$

Now (12) implies that $f_{g}(t)=h(t)$ for all $t$ in $\left(-\infty_{0} 0\right]$, for if $p(t)=$ $\left|f_{g}(t)-h(t)\right|$ for $t \in(-\infty, 0]$, then $p$ has a left derivative on $(-\infty, 0]$ and $p_{-}^{\prime}(t)=m_{-}\left[f_{g}(t)-h(t), f_{g}^{\prime}(t)-h^{\prime}(t)\right]$ (see Coppel [1, p. 3]). Thus, by (8) and (12),

$$
p^{\prime}(t)=: m\left[f_{!}(t)-h(t), G\left[f_{g}\right](t)-G[h](t)\right] \leqq-p(t) \leqq 0 ;
$$

so $p$ is nonincreasing and, since $\lim _{t \rightarrow-} p(t)=0$, it follows that $p(t)=0$ for all $t \cdots(-\infty, 0]$. In particular $f_{y}(0)=h(0)=z$, and by (11) and Lemma 1 the function $u_{\text {. }}$ defined on $[0, b]$ by $u_{z}(t)=e^{\gamma t} f_{g}(t)$ satisfies $(2)$ and (3) for $t$ in $[0, b]$. Since $b$ can be any positive number, the assertions of the Theorem are seen to be true. 


\section{REFERENCFS}

1. W. A. Coppel, Stability and asymptotic behavior of differential equations, D. C. Heath, Boston, Mass., 1965. MR 32 \#7875.

2. J. Dieudonné, Foundations of modern analysis, Pure and Appl. Math., vol. 10, Academic Press, New York, 1960. MR 22 \#11074.

3. J. V. Herod, A pairing of a class of evolution systems with a class of generators, Trans. Amer. Math. Soc. 157 (1971), 247-260.

4. E. Hille and R. S. Phillips, Functional analysis and semi-groups, rev. ed., Amer. Math. Soc. Colloq. Publ., vol. 31, Amer. Math. Soc., Providence, R.I., 1957. MR 19, 664.

5. D. L. Lovelady, A functional differential equation in a Banach space, Funk. Ekvac. 14 (1971), 111-122.

6. R. H. Martin, Jr., A global existence theorem for autonomous differential equations in a Banach space, Proc. Amer. Math. Soc. 26 (1970), 307-314. MR 41 \#8791.

7. - Differential equations on closed subsets of a Banach space, Trans. Amer. Math. Soc. (to appear).

8. G. F. Webb, Nonlinear evolution equations and product stable operators on Banach spaces, Trans. Amer. Math. Soc. 155 (1971), 409-426.

9. - Product integral representation of time dependent nonlinear evolution equations in Banach spaces, Pacific J. Math. 32 (1970), 269-281.

10. - Continuous nonlinear perturbations of linear accretive operators in Banach spaces, J. Functional Analysis (to appear).

Department of Mathematics, University of South Carolina, Columbia, South CARolina 29208

Department of Mathematics, North Carolina State University, Raleigh, North Carolina 27607 (Current address of Robert H. Martin, Jr.)

Current address (David Lowell Lovelady): Department of Mathematics, Florida State University, Tallahassee, Florida 32306 